\title{
Low energy monopole Modes of a Trapped atomic Fermi Gas
}

\author{
G. M. Bruun* \\ Nordita, Blegdamsvej 17, 2100 Copenhagen, Denmark
}

We consider the low energy collective monopole modes of a trapped weakly interacting atomic Fermi gas in the collisionless regime. The spectrum is calculated for varying coupling strength and chemical potential. Using an effective Hamiltonian, we derive analytical results that agree well with numerical calculations in various regimes. The onset of superfluidity is shown to lead to effects such as the vanishing of the energy required to create a Cooper molecule at a critical coupling strength and to the emergence of pair vibration excitations. Our analysis suggests ways to experimentally detect the presence of the superfluid phase in trapped atomic Fermi gases.

Pacs Numbers: 67., 05.30.Fk, 32.80.Pj

The trapping and cooling of fermionic atoms is attracting increasing attention in the field of ultra cold atomic gases. Temperatures as low as $T \sim 0.2 T_{F}$ with $T_{F}$ denoting the Fermi temperature have recently been observed experimentally for such gases [1]. One of the intriguing goals of the impressive experimental effort is to observe the theoretically predicted transition to a superfluid state below a critical temperature $T_{c}$ when the effective atomatom interaction is attractive [2].

In this paper, the $T=0$ low energy collective monopole modes of a weakly interacting fermi gas in the collisionless limit are examined both analytically and numerically. We identify spectral features characteristic of the onset of superfluidity. Since the collective modes of trapped gases can be measured with high precision [3], this leads us to suggest appealing ways of experimentally observing the predicted phase-transition.

Consider a dilute gas of fermionic atoms of mass $m$ with an equal number of atoms $N_{\sigma}$ in two hyperfine states $|\sigma=\uparrow, \downarrow\rangle$ trapped in a spherically symmetric harmonic potential of the form $U_{0}(r)=m \omega_{T}^{2} r^{2} / 2$. Assuming that the gas is in the dilute regime $k_{F}|a| \ll 1$ with $k_{F}=\sqrt{2 m \mu_{F}}\left(\mu_{F}\right.$ is the chemical potential $)$, the interaction between atoms in the two different hyperfine states can be described as $g \delta(\mathbf{r})$ where $g=4 \pi a / m$ and $a$ is the $s$-wave scattering length. We put $\hbar=1$ throughout the paper. For $a<0$ the effective atom-atom interaction is attractive and the gas is unstable toward the formation of Cooper pairs below a certain critical temperature $T_{c}$ [2]. The quasiparticle excitations of the superfluid state can be calculated by solving the Bogoliubov-de Gennes (BdG) equations [4].

The collective excitations of the gas can be calculated in the collisionless limit using the random-phase approximation (RPA). In a real space formalism, the poles of the matrix

$$
\Pi(\omega)=\left\{\begin{array}{cccc}
\left\langle\left\langle\hat{\rho}_{\uparrow} \hat{\rho}_{\uparrow}\right\rangle\right\rangle & \left\langle\left\langle\hat{\rho}_{\uparrow} \hat{\rho}_{\downarrow}\right\rangle\right\rangle & \left\langle\left\langle\hat{\rho}_{\uparrow} \hat{\chi}\right\rangle\right\rangle & \left\langle\left\langle\hat{\rho}_{\uparrow} \hat{\chi}^{\dagger}\right\rangle\right\rangle \\
\left\langle\left\langle\hat{\rho}_{\downarrow} \hat{\rho}_{\uparrow}\right\rangle\right\rangle & \left\langle\left\langle\hat{\rho}_{\downarrow} \hat{\rho}_{\downarrow}\right\rangle\right\rangle & \left\langle\left\langle\hat{\rho}_{\downarrow} \hat{\chi}\right\rangle\right\rangle & \left\langle\left\langle\hat{\rho}_{\downarrow} \hat{\chi}^{\dagger}\right\rangle\right\rangle \\
\left\langle\left\langle\hat{\chi} \hat{\rho}_{\uparrow}\right\rangle\right\rangle & \left\langle\left\langle\hat{\chi} \hat{\rho}_{\downarrow}\right\rangle\right\rangle & \langle\langle\hat{\chi} \hat{\chi}\rangle\rangle & \left\langle\left\langle\hat{\chi} \hat{\chi}^{\dagger}\right\rangle\right\rangle \\
\left\langle\left\langle\hat{\chi}^{\dagger} \hat{\rho}_{\uparrow}\right\rangle\right\rangle & \left\langle\left\langle\hat{\chi}^{\dagger} \hat{\rho}_{\downarrow}\right\rangle\right\rangle & \left\langle\left\langle\hat{\chi}^{\dagger} \hat{\chi}\right\rangle\right\rangle & \left\langle\left\langle\hat{\chi}^{\dagger} \hat{\chi}^{\dagger}\right\rangle\right\rangle
\end{array}\right\}
$$

yield the modes of the system. Here, $\langle\langle\hat{A} \hat{B}\rangle\rangle$ is the Fourier transform of the retarded function $-i \Theta(t-$ $\left.t^{\prime}\right)\left\langle\left[\hat{A}(\mathbf{r}, t), \hat{B}\left(\mathbf{r}^{\prime}, t^{\prime}\right)\right]\right\rangle$ and $\langle\ldots\rangle$ is the thermal average. Also, $\hat{\rho}_{\sigma}(\mathbf{r})=\hat{\psi}_{\sigma}^{\dagger}(\mathbf{r}) \hat{\psi}_{\sigma}(\mathbf{r})$ and $\hat{\chi}(\mathbf{r})=\hat{\psi}_{\downarrow}(\mathbf{r}) \hat{\psi}_{\uparrow}(\mathbf{r})$. Correlation functions with the operator $\hat{\chi}(\mathbf{r})$ are included in order to describe the effects of superfluidity. A numerical scheme for calculating $\Pi(\omega)$ within the RPA was described in Ref. [5].

The nature of the superfluid state depends on two parameters: the number of particles trapped and the interaction strength [6]. When the atom-atom interaction is sufficiently weak and/or there are not too many particles trapped, the gas is in the so-called intrashell regime; it can in many ways best be regarded as a giant nucleus. In this regime, the Cooper pairs are formed only between particles with angular quantum numbers $(l, m)$ and $(l,-m)$ residing in the same harmonic oscillator shell with radial quantum number $n$ [i.e. single particle energy $\left.\epsilon_{n}=(n+3 / 2) \omega_{T}\right]$. For stronger interactions and/or more particles trapped such that $\xi_{B C S} \ll R$ where $\xi_{B C S}=k_{F}(\pi m \Delta)^{-1}$ is the coherence length $(\Delta$ is the pairing gap) and $R$ is the size of the system given by the Thomas-fermi result $R \simeq \sqrt{2 \mu_{F} / m}$, the superfluid state is best described as a quasi-homogenous bulk system. The transition between the two qualitatively different regimes is roughly determined by $\xi_{B C S} \simeq R$ or equivalently $\Delta \simeq \omega_{T}$. Using the bulk theory prediction $\Delta=$ $(2 / e)^{7 / 3} \mu_{F} \exp \left(-\pi / 2 k_{F}|a|\right)[8]$ and the Thomas-Fermi result $\mu_{F}=\left(6 N_{\sigma}\right)^{1 / 3} \omega_{T}$ (neglecting small effects due to the pairing as $\Delta / \mu_{F} \ll 1$ in the dilute regime), the equation $\Delta=\omega_{T}$ yields $N_{\sigma}^{*}=\frac{1}{6}\left(\frac{e}{2}\right)^{7} \exp \left(3 \pi / 2 k_{F}|a|\right)$. For a given coupling strength with $N_{\sigma} \ll N_{\sigma}^{*}$ atoms trapped, the gas is in the in the intrashell regime and for $N_{\sigma} \gg N_{\sigma}^{*}$ atoms trapped it is in the bulk regime. With $k_{F}|a|=0.1$ this condition yields $N_{\sigma}^{*}=4 \times 10^{20}$ and for $k_{F}|a|=0.3$ we get $N_{\sigma}^{*}=9 \times 10^{6}$. Current experiments have $N \sim \mathcal{O}\left(10^{5}\right)$ particles trapped [1]. Also, the present experimental effort uses optical traps as the confining potential as they allow greater greater flexibility with respect to which atomic hyperfine states can be trapped [1]. The optical traps used so far have $1.2 \mathrm{kHZ} \leq \omega_{T} / 2 \pi \leq 12 \mathrm{kHZ}$ corresponding to temperatures $58 \mathrm{nK} \leq T \leq 580 \mathrm{nK}$ [1]. Thus, the condition $k_{B} T_{c} \ll \omega_{T}$ is less restrictive than for magnetic traps. Note also that $k_{B} T_{c} \simeq \Delta(T=0) / 2$ in the intrashell regime can be orders of magnitude higher than 
the bulk prediction [9]. The above estimates seem to indicate, that for the gas in the dilute limit it is not unlikely that future experiments on trapped superfluid fermionic atoms will be in the intrashell regime.

For $k_{F}|a| \gtrsim 0.3$ the gas is not in the dilute limit. Recently, a number of papers have been dealing with the case of a Feshbach resonance mediated interaction, which is predicted to increase $T_{c}$ dramatically [10]. This could make the experimental observation of the superfluid transition easier. It is however presently unclear what happens in this strongly correlated regime where issues such as the instability toward spinodal decomposition have to be addressed.

The collective modes also depends qualitatively on which regime the gas is in. In the bulk regime, the modes can be calculated by looking at phase fluctuations of the pairing field. A gradient and frequency expansion of the lower right $2 \times 2$ part of Eq. (1) then yields a hydrodynamic spectrum for the lowest modes of the gas [7].

In light of the possible experimental relevance, we consider in this paper the collective modes in the intrashell regime. From the fact that there is only pairing between particles with quantum numbers $(n, l, m)$ and $(n, l,-m)$ follows a crucial simplification: Ignoring for the time being the effect of the Hartree field, the monopole pairing correlations of the particles are accurately described by the effective Hamiltonian

$$
\begin{gathered}
\hat{H}_{e f f}=\sum_{n l m \sigma} \xi_{n} \hat{a}_{n l m \sigma}^{\dagger} \hat{a}_{n l m \sigma}- \\
\frac{2 G \omega_{T}}{\Omega_{n_{F}}} \sum_{n l m, n^{\prime} l^{\prime} m^{\prime}}(-1)^{m+m^{\prime}} \hat{a}_{n l m \uparrow}^{\dagger} \hat{a}_{n l-m \downarrow}^{\dagger} \hat{a}_{n^{\prime} l^{\prime}-m^{\prime} \downarrow} \hat{a}_{n^{\prime} l^{\prime} m^{\prime} \uparrow}(2)
\end{gathered}
$$

in the sense that it can be shown that Eq. (2) reproduces the gap equation and thus the results concerning the quasiparticle properties presented in Ref. [6] in the intrashell regime. Here $G=32 \pi^{-2} k_{F}|a| / 15$ is the effective coupling strength and $\Omega_{n_{F}}=\left(n_{F}+1\right)\left(n_{F}+2\right) / 2$ is the pair degeneracy of the highest harmonic oscillator level occupied with radial quantum number $n_{F}$. The operator $\hat{a}_{n l m \sigma}^{\dagger}$ creates a particle with quantum numbers $(n, l, m, \sigma)$ and energy $\xi_{n}=\epsilon_{n}-\mu_{F}$. This effective Hamiltonian is much simpler to work with than the full Hamiltonian and it allows several analytic results to be derived. It should be emphasized that Eq. (2) can be derived from the full Hamiltonian in the intrashell regime; it thus describes the actual physical system accurately and is not merely a schematic model as we shall see.

In order to derive analytical results, we write the RPA eqn. for $T=0$ in configuration space as [11]

$$
\left\{\begin{array}{cc}
A & B \\
-B^{*} & -A^{*}
\end{array}\right\}\left\{\begin{array}{c}
X^{\nu} \\
Y^{\nu}
\end{array}\right\}=\omega_{\nu}\left\{\begin{array}{c}
X^{\nu} \\
Y^{\nu}
\end{array}\right\} .
$$

Here

$\omega_{\nu}$

the frequency of the excitation $|0\rangle \rightarrow|\nu\rangle=\hat{\Gamma}_{\nu}^{\dagger}|0\rangle$ where $|0\rangle$ and $|\nu\rangle$ is the RPA ground and excited state respectively. The matrices $A$ and $B$ are given by $A_{n l m, n^{\prime} l^{\prime} m^{\prime}}=$ $\left\langle B C S\left|\hat{\gamma}_{n l-m \downarrow} \hat{\gamma}_{n l m \uparrow} \hat{H} \hat{\gamma}_{n^{\prime} l^{\prime} m^{\prime} \uparrow}^{\dagger} \hat{\gamma}_{n^{\prime} l^{\prime}-m^{\prime} \downarrow}^{\dagger}\right| B C S\right\rangle$

and

$B_{n l m, n^{\prime} l^{\prime} m^{\prime}}$

$\left\langle B C S\left|\hat{\gamma}_{n l-m \downarrow} \hat{\gamma}_{n l m \uparrow \uparrow} \hat{\gamma}_{n^{\prime} l^{\prime}-m^{\prime} \downarrow} \hat{\gamma}_{n^{\prime} l^{\prime} m^{\prime} \uparrow} \hat{H}\right| B C S\right\rangle . \quad$ The superfluid ground state $|B C S\rangle$ and the quasi particle creation operator $\hat{\gamma}_{n l m \sigma}^{\dagger}$ with quantum numbers $(n, l, m, \sigma)$ can both can be obtained from a solution to the BdG equations [4]. Also, $X_{n l m}=\left\langle 0\left|\hat{\gamma}_{n l-m \downarrow} \hat{\gamma}_{n l m \uparrow}\right| \nu\right\rangle$ and $Y_{n l m}=\left\langle 0\left|\hat{\gamma}_{n l m \uparrow}^{\dagger} \hat{\gamma}_{n l-m \downarrow}^{\dagger}\right| \nu\right\rangle$. In the intrashell regime, $\hat{\gamma}_{n l m \sigma}=u_{n} \hat{a}_{n l m \sigma} \pm v_{n} \hat{a}_{n l-m-\sigma}^{\dagger}(-1)^{m}, \quad u_{n}^{2}=(1+$ $\left.\xi_{n} / E_{n}\right) / 2$ and $v_{n}^{2}=\left(1-\xi_{n} / E_{n}\right) / 2$ with $E_{n}=\left(\xi_{n}^{2}+\right.$ $\left.\Delta^{2}\right)^{1 / 2}[6]$. We have written the RPA equations appropriate for subspace spanned by monopole pairing excitations of the kind $\hat{\gamma}_{n l m \uparrow}^{\dagger} \hat{\gamma}_{n l-m \downarrow}^{\dagger}+$ c.c.

We first discuss the solutions to Eq. (3) when the gas is in the normal phase $(\Delta=0)$. Excitations of monopole symmetry are given by $\hat{\Gamma}_{\nu}^{\dagger}=$ $\sum_{n l m}(-1)^{m}\left[X_{n}^{\nu} \hat{\gamma}_{n l m \uparrow}^{\dagger} \hat{\gamma}_{n l-m \downarrow}^{\dagger}+Y_{n}^{\nu} \hat{\gamma}_{n l-m \downarrow} \hat{\gamma}_{n l m \uparrow}\right]$, i.e. $X_{n l m}^{\nu}=(-1)^{m} X_{n}^{\nu}$ and $Y_{n l m}^{\nu}=(-1)^{m} Y_{n}^{\nu}$ in Eq. (3). Using this we obtain after some algebra

$$
\frac{1}{2 G}=\sum_{n>n_{F}} \frac{1}{\omega+2 \xi_{n}}-\sum_{n<n_{F}} \frac{1}{\omega+2 \xi_{n}} .
$$

Equation (4) determines the lowest collective monopole modes of the gas in the normal phase. These modes are not

ticle conserving. They correspond to adding/removing a pair of particles; i.e. one obtains for the addition mode $\hat{\Gamma}_{a}^{\dagger}=\sum_{n>n_{F} l m}(-1)^{m} X_{n} \hat{a}_{n l m \uparrow}^{\dagger} \hat{a}_{n l-m \downarrow}^{\dagger}+$ $\sum_{n<n_{F} l m}(-1)^{m} Y_{n} \hat{a}_{n l m \uparrow}^{\dagger} \hat{a}_{n l-m \downarrow}^{\dagger}$ with energy $\omega_{a}$ and likewise for the removal mode. The lowest particle conserving monopole excitation is then given by the state $\hat{\Gamma}_{r}^{\dagger} \hat{\Gamma}_{a}^{\dagger}|0\rangle$ with energy $\omega_{r}+\omega_{a}$.

Consider the case $\mu_{F}=\left(n_{F}+2\right) \omega_{T}$ corresponding to a symmetric level distribution around the Fermi level with levels below $\mu_{F}$ (i.e. $n \leq n_{F}$ ) completely filled. In this case, the system is in the normal phase for $T=0$ for weak coupling strengths such that $G<G_{c} . G_{c}$ is obtained by setting $\Delta=0$ in the gap equation reading $G^{-1}-$ $\sum_{n} E_{n}^{-1}=0$. This yields $G_{c}^{-1} \sim 2^{5 / 2} \ln \left[4 \exp (\gamma) n_{F}\right] / 3$ where $\gamma \simeq 0.577$ is Eulers constant [6]. Note that this equation is reproduced by setting $\omega=0$ in Eq. (4). We therefore conclude that the closed shell system becomes unstable toward the formation of Cooper pairs when the energy cost of adding/removing a pair of particles goes to zero. Equation (4) can be solved for $G-G_{c} \rightarrow 0_{-}$ yielding

$$
\frac{\omega_{r}+\omega_{a}}{\omega_{T}}=2\left[\frac{2}{7 \xi(3)}\right]^{1 / 2} \sqrt{\frac{1}{G}-\frac{1}{G_{c}}}, \quad G-G_{c} \rightarrow 0_{-}
$$

with $\xi(3)=\sum_{n=1}^{\infty} n^{-3} \simeq 1.202$. This equation gives the frequency of the lowest particle conserving monopole mode as the closed shell system becomes unstable toward the formation of a superfluid with increasing coupling 
strength. For $G \rightarrow 0$, we can also solve Eq. (4) obtaining

$$
\left(\omega_{r}+\omega_{a}\right) / \omega_{T}=2-4 G, \quad G \rightarrow 0 .
$$

We
$\sum_{l m, l m^{\prime}}(-1)^{m+m^{\prime}} \hat{a}_{n_{F}+1 l m \uparrow}^{\dagger} \hat{a}_{n_{F}+1 l-m \downarrow}^{\dagger} \hat{\Gamma}_{a}^{\dagger} \hat{\Gamma}_{r}^{\dagger}$$\rightarrow$ for $G \rightarrow 0$; i.e. we simply take a pair of particles in the highest filled shell and put them into a monopole state in the lowest empty shell. This obviously costs $2 \omega_{T}$ in energy for $G \rightarrow 0$. Equation (6) can also be obtained by a first order perturbative calculation of the first excited state energy [12]. Equation (4-6) give the lowest monopole excitation for a closed shell system in the normal phase.

We now solve Eq. (3) when the gas is in the superfluid phase $(\Delta>0)$. Using $X_{n l m}=(-1)^{m} X_{n}$ and $Y_{n l m}=$ $(-1)^{m} Y_{n}$, we obtain after some rather lengthy algebra:

$$
\left|\begin{array}{cc}
G^{-1}+4 \sum_{n} \frac{E_{n}}{\omega^{2}-4 E_{n}^{2}} & 2 \omega \sum_{n} \frac{\xi_{n}}{E_{n}\left(\omega^{2}-4 E^{2}\right)} \\
2 \omega \sum_{n} \frac{\xi_{n}}{E_{n}\left(\omega^{2}-4 E_{n}^{2}\right)} & G^{-1}+4 \sum_{n} \frac{\xi_{n}^{2}}{E_{n}\left(\omega^{2}-4 E_{n}^{2}\right)}
\end{array}\right|=0 .
$$

Equation (7) determines the frequencies $\omega$ of the lowest monopole pair vibrations of the gas in the superfluid phase. First, we note that it follows from the gap equation that $\omega=0$ is a solution to Eq. (7). This is the spurious solution coming from the fact that BCS theory breaks the $U(1)$ gauge symmetry of the pair-field or equivalently particle conservation. We now examine the two opposite cases of interest: The completely filled and the open shell case.

First consider the case of $\mu_{F}=\left(n_{F}+2\right) \omega_{T}$ with $G>$ $G_{c}$ such that the gas is superfluid. The solutions to Eq. (7) split into an even solution $\left(X_{n_{F}+n}=X_{n_{F}-n}\right.$ and $Y_{n_{F}+n}=Y_{n_{F}-n}$ ) corresponding to the spurious mode with $\omega=0$ and an odd solution with $X_{n_{F}+n}=-X_{n_{F}-n}$ and $Y_{n_{F}+n}=-Y_{n_{F}-n}$. Some algebra yields the odd solution energy as

$$
\frac{\omega}{\omega_{T}}=\frac{2 \Delta}{\omega_{T}}=\left[\frac{2^{1 / 2} 3}{7 \xi(3)}\right]^{1 / 2} \sqrt{\frac{1}{G_{c}}-\frac{1}{G}}, \quad G-G_{c} \rightarrow 0_{+}
$$

where we have used $\Delta=\left[2^{-3 / 2} 7^{-1} \xi(3)^{-1} 3\right]^{1 / 2}\left(G_{c}^{-1}-\right.$ $\left.G^{-1}\right)^{1 / 2}$ for $G>G_{c}$ in the closed shell case [6]. This is the pair vibration mode $\hat{\Gamma}_{P}^{\dagger}|0\rangle$ where the modulus $|\Delta|$ of $\Delta$ oscillates. Note that $2 \Delta$ is smaller than the lowest particle conserving quasiparticle excitation energy: $2 E_{n_{F}}=2 \sqrt{\xi_{n_{f}}^{2}+\Delta^{2}}>2 \Delta\left(\xi_{n_{F}}=-\omega_{T} / 2\right)$. The pair vibration mode corresponds to coherent excitations of quasiparticle pairs of monopole symmetry such that the resulting energy is smaller than $2 E_{n_{F}}$; the mode is strongly collective. It also conserves the number of particles and can be excited by a particle conserving probe of monopole symmetry. We conclude that in the case of a closed shell configuration, one can detect experimentally the onset of superfluidity with increasing coupling strength by observing the lowest monopole excitation frequency of the gas vanish as given by Eq. (5). The system becomes increasingly unstable toward the excitation of pairs of particles into the lowest empty band as they can use the degeneracy of this band to maximize their spatial overlap and thereby increase the (absolute value of) interaction energy; the energy cost of forming a Cooper molecule simply goes to zero at the critical coupling strength. Once the system is superfluid for $G>G_{c}$, the lowest monopole excitation energy again increases as given by Eq. (8). Now the ground state of the system already consist of correlated pair excitations across the Fermi level to create Cooper pairs with maximum spatial overlap and the lowest excitation is the pair vibration.

In the case of an open shell with $\mu_{F}=\left(n_{F}+3 / 2\right) \omega_{T}$, the gas remains superfluid for $G \rightarrow 0_{+}$for $T=0$. In this limit, there is only pairing in the shell at the chemical potential $\left(n=n_{F}\right)$ [6]. Solving the RPA equations for this shell, we obtain the $\omega=0$ spurious solution and the pair breaking solution with energy $2 E_{n_{F}}=2 \Delta$ $\left(\xi_{n_{F}}=0\right)$. Residual interactions between between the excitations lower the frequency slightly below $2 \Delta$. To a good approximation however, the lowest monopole excitation energy of the system is

$$
\frac{\omega}{\omega_{T}} \simeq \frac{2 \Delta}{\omega_{T}}=\frac{2 G}{1-2^{5 / 2} \ln \left(e^{\gamma} n_{F}\right) G / 3}, \quad G \rightarrow 0_{+}
$$

where

we have used $\Delta / \omega_{T}=G\left[1-2^{5 / 2} \ln \left(\exp (\gamma) n_{F}\right) G / 3\right]^{-1}$ in the open shell case [6]. Superfluidity can thus also in the open shell case be detected by the presence of a low energy $\omega \simeq 2 \Delta$ mode.

We now compare our analytical results with an exact numerical solution of the RPA equations obtained using the method described in Ref. [5]. First, consider the case of a closed shell system $\left[\mu_{F}=\left(n_{F}+2\right) \omega_{T}\right]$. In fig. 1 (a) we plot the lowest monopole excitation energy as a function of the coupling strength $G / G_{c}$. For $G<G_{c}$ the excitation is $\hat{\Gamma}_{r}^{\dagger} \hat{\Gamma}_{a}^{\dagger}|0\rangle$ whereas for $G>G_{c}$ the excitation is $\hat{\Gamma}_{P}^{\dagger}|0\rangle$. The $\times$ 's are obtained from a numerical calculation of the matrix given in Eq. (1) and the lines are Eq. (5), (6), and (8). We have chosen $n_{F}=50$ corresponding to $\sim 2 \times 10^{4}$ particles trapped in each of the two hyperfine states. There is very good agreement between the numerical results and the analytical predictions in their regions of validity. This shows that effective Hamiltonian given by Eq. (2) accurately describes the pairing correlations for the physical system in the intrashell regime. As the coupling strength increases, the intrashell pairing ansatz eventually breaks down; this occurs for $G / G_{c} \sim 1.2$ for this set of parameters [6]. For stronger coupling, Eq. (2) is invalid and one needs to work with the full Hamiltonian and Eq. (1). This break down is seen numerically as a fragmentation and disappearance of the $\omega \sim 2 \Delta$ pair vibration mode for $G / G_{c} \sim 1.2$. Eventually, when $\xi_{B C S} \ll R$ or equivalently $G \gg G_{c}$, the lowest collective excitations are not the pair vibrations corresponding to fluctuations in $|\Delta|$ but the Goldstone modes correspond- 
ing to phase fluctuations of $\Delta[5,7]$. The lowest monopole mode is $\omega=2 \omega_{T}$ in this regime.

Let us discuss the open shell case $\mu_{F}=(n+3 / 2) \omega_{T}$ with $n_{F}=50$. In fig. 1 (b), we plot the lowest monopole excitation energy as a function of the coupling strength $G / \omega_{T}$. The $\times$ 's are obtained from a numerical calculation of the matrix given in Eq. (1) and the line is Eq. (9). We see that there is good agreement between the numerical results and Eq. (9) for $G \rightarrow 0$. This shows that in the open shell case, the lowest excitations are essentially quasi-particle excitations with energy $2 \Delta$ in the intrashell regime. The exact mode frequency is somewhat lower than $2 \Delta$ as expected reflecting the residual interaction between the quasiparticles. With increasing coupling, the intrashell eventually ansatz breaks down for $G \ln \left(N_{F}\right) \sim 0.4$ [6] making Eq. (2) and thus Eq. (9) invalid. Again, for stronger coupling when $\xi_{B C S} \ll R$ the Goldstone modes are the lowest excitations.

Several other methods to observe the superfluid transition for atomic Fermi gases have been suggested in the literature. They include laser probing of the pairing energy, looking at the expansion properties of the gas, and probing the density profile [13].

We have up till now ignored the effects of the Hartree field. This field introduces a dispersion on the Harmonic oscillator levels with respect to the angular momentum $l$ lifting the shell degeneracy $\Omega_{n}=(n+1)(n+2) / 2$. One should therefore replace $\xi_{n}$ with an $l$-dependent $\tilde{\xi}_{n l}$ in Eq. (2), were $\tilde{\xi}_{n l}$ includes the effect of the Hartree field on the single particle energies. The resulting effective Hamiltonian describes the pairing correlations in the intrashell regime including the effects of the Hartree field. When the splitting introduced by the Hartree field is small compared with the pairing energy $\Delta$, it can be ignored. This is the case for systems with $n_{F} \lesssim 30$ (depending on the coupling strength) [6]. For very large systems with $n_{F} \gg 200$, there is only pairing within the levels with $\tilde{\xi}_{n l}=0$ and $m=-l,-l+1 \ldots, l$. An analysis completely similar to the one given above yields the lowest excitation energy $\omega \simeq 2 \Delta_{n_{F} l}$ in this limit with $\Delta_{n_{F} l}$ denoting the pairing energy in the level $\tilde{\xi}_{n l}=0$. We plot in fig. 1 (b) as +'s the results for lowest monopole excitation energy obtained from a numerical calculation based on Eq. (1) including the effects of the Hartree field. For comparison with the results obtained neglecting the Hartree field, the number of particles is fixed in both cases. We see that for $G \rightarrow 0$, the Hartree field suppresses the pairing energy as it introduces a splitting of the levels in the shell at the chemical potential such that the density of states for $\tilde{\xi}_{n l}=0$ is reduced. For stronger coupling however, the Hartree field increases the pairing energy. This is because the Hartree field increases the density and thus the spatial overlap between particles in the center of trap [6]. From fig. 1 (b), we see that Eq. (9) reproduces qualitatively the energy of the lowest mode as a function of $G$ even when the Hartree field is included. This is as expected since $n_{F}=50$ is an inter- mediate value where the pairing is not in the limit where the Hartree field can be ignored, but also far away from the regime, where the Hartree splitting is much larger than the pairing energy.

It should be noted that the conclusion that there are pair vibration modes with an energy $\sim 2 \Delta$ is independent of the symmetry of the trapping potential. This result simply reflects the energy required to break individual Cooper pairs. However, the exact value of $\Delta$ as a function of the coupling strength depends on the symmetry of the potential and Eq. (5-6), (8-9) are of course only valid for a spherical trap.

In summary, we examined in detail the low energy monopole modes of a trapped fermi gas as a function of the coupling strength and the chemical potential. Using an effective Hamiltonian describing the pair correlations in the intrashell regime we derived analytical results that agree well with numerical calculations. As the gas approaches the superfluid instability with increasing coupling strength for the closed shell case, the lowest excitation energy corresponding to the creation of a Cooper molecule was shown to vanish. In the superfluid phase, the lowest excitation energy is $\omega \simeq 2 \Delta$ independent of the position of the chemical potential. The results presented should be relevant for the present experimental effort to observe the superfluid transition.

We acknowledge valuable discussions with B. R. Mottelson.

* Present address: Niels Bohr Institute, Blegdamsvej 17, 2100 Copenhagen, Denmark

[1] T. Loftus et al., Phys. Rev. Lett. 88, 173201 (2002); A. G. Truscott et al., Science 291, 2570 (2001); F. Schreck et al, Phys. Rev. A 64, 011402 (2001); S. R. Granada et al., Phys. Rev. Lett. 88, 120405 (2002); K. Dieckmann et al., cond-mat/0207046

[2] H. T. C. Stoof, M. Houbiers, C. A. Sackett, and R. G. Hulet, Phys. Rev. Lett. 76, 10 (1996)

[3] See e.g. F. Dalfovo, S. Giorgini, L. P. Pitaevskii, and S. Stringari, Rev. Mod. Phys. 71, 463 (1999)

[4] G. M. Bruun, Y. Castin, R. Dum, and K. Burnett, Eur. Phys. J. D 9, 433 (1999)

[5] G. M. Bruun and B. R. Mottelson, Phys. Rev. Lett. 87, 270403 (2001)

[6] G. M. Bruun and H. Heiselberg, Phys. Rev. A 65, 053407 (2002); H. Heiselberg and B. R. Mottelson, condmat/0112248

[7] M. A. Baranov and D. S. Petrov, Phys. Rev. A 62, 041601 (2000)

[8] L. P. Gorkov and T. K. Melik-Barkhudarov, Sov. Phys. JETP 13, 1018 (1961); H. Heiselberg et al. Phys. Rev. Lett. 85, 2418 (2000)

[9] G. M. Bruun, Phys. Rev. A 66, 041602(R) (2002).

[10] M. Holland et al., Phys. Rev. Lett. 87, 120406 (2001); 
E. Timmermans, Phys. Lett. 285, 228 (2001); Y. Ohashi and A. Griffin, Phys. Rev. Lett. 89, 130402 (2002); S. J. J. M. F. Kokkelmans, J. N. Milstein, M. L. Chiofalo, R. Walser, and M. J. Holland, Phys. Rev. A 65, 053617 (2002).

[11] J. P. Blaizot and G. Ripka, Quantum Theory of Finite Systems (MIT press, Cambridge Mass., 1986)

[12] J. Högaasen-Feldman, Nucl. Phys. 28, 258 (1961)

[13] P. Törma and P. Zoller, Phys. Rev. Lett. 85, 487 (2000); G. M. Bruun, P. Törma, M. Rodriguez, and P. Zoller, Phys. Rev. A 64, 033609 (2001); C. Menotti, P. Pedri, and S. Stringari cond-mat/0208150; M. L. Chiofalo, S. J. J. M. F. Kokkelmans, J. N. Milstein, and M. J. Holland, Phys. Rev. Lett. 88, 090402 (2002).

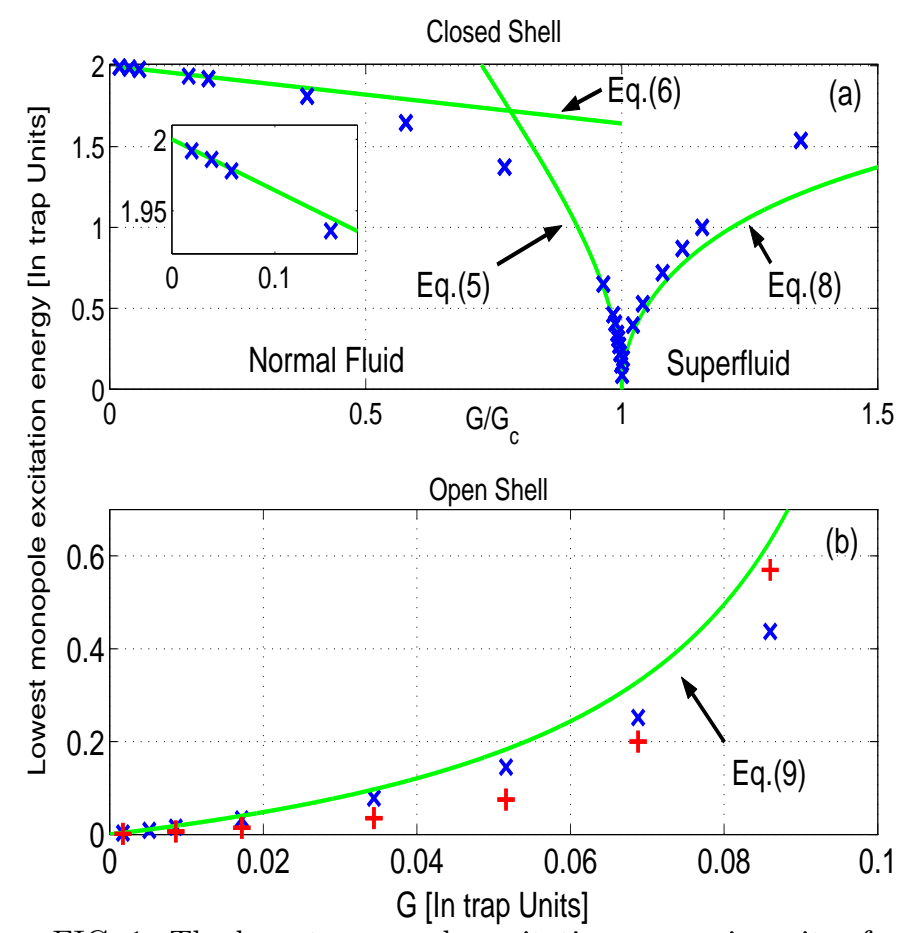

FIG. 1. The lowest monopole excitation energy in units of $\omega_{T}$ for (a): a closed shell system as a function of $G / G_{c}$ and (b): an open shell system as a function of $G / \omega_{T}$. The inset in (a) shows the $G \rightarrow 0$ region in detail. 